\title{
Evaluation of a novel communication and consultation skills model (WISE COACH) on dog owner perceptions of veterinarians and projected spending on veterinary care
}

\author{
James J. Clark, DVM, MBA, * and Cassandra M. Linder, DVM \\ Dean's Office, School of Veterinary Medicine, University of California-Davis, Davis, CA \\ ${ }^{*}$ Corresponding author: Dr. Clark (jclark@ucdavis.edu) \\ https://doi.org/10.2460/javma.21.02.0096
}

\section{OBJECTIVE}

To assess the impact of a novel communication and consultation skills model (WISE COACH [WC]) on dog owner perceptions of veterinarians and projected spending on veterinary care.

\section{SAMPLE}

1,200 US dog owners who had visited a veterinarian within the prior 18 months.

\section{PROCEDURES}

Video recordings of 2 staged client consultations were made, with the veterinarian following the WC recommendations in one video and not following them in the other (control). Participants were randomly assigned to view one of the videos and completed an online survey to assess their perceptions and projected spending. Qualitative responses were coded to identify themes.

\section{RESULTS}

The veterinarian was rated significantly higher in the WC video than in the control video for the characteristics first impression, skilled and knowledgeable, cares about me, cares about my pet, and communicates clearly, and was rated significantly lower for the characteristic rushed or abrupt. Participants who viewed the WC video were significantly more likely to follow the veterinarian's recommendations, return to see the veterinarian, and recommend the veterinarian. They were also approximately 1.4 times as likely to approve the full recommended treatment plan, and their projected total spending was approximately 15\% higher than projected spending for participants who viewed the control video.

\section{CLINICAL RELEVANCE}

Results showed improved client perceptions, client retention, quality of patient care, and financial metrics when the veterinarian followed the WC recommendations. Further study is needed to determine whether this model may also improve veterinarian well-being by improving client relationships and decreasing resistance to recommendations.

$\mathrm{Cl}$ ient consultations are among the most frequenty performed tasks in veterinary clinical practice, ${ }^{1}$ and while there is a growing body of literature on client communication, relatively little research has been conducted within the profession to provide guidance on approaches to the complex task of conducting effective client consultations. In a previous study, ${ }^{2}$ median duration of a typical office visit was only 9 to 10 minutes, but during this time, the veterinarian must accomplish numerous tasks, such as establishing a relationship with the client, collecting a history and performing a physical examination, educating the client about the risks and benefits of treatment, and discussing potential costs. Additionally, to obtain informed consent in an ethical manner and protect themselves and their practices legally, veterinarians must cover certain specific content during a client consultation. In human medicine, a relationship-centered strategy with evidence-based guidelines for patient communication has been documented to improve the patient experience and outcomes, ${ }^{3}$ but no similar veterinary-specific framework has been validated for common use.

Developing a veterinary-specific consultation framework that includes evidence-based guidelines for effective communication would likely benefit the veterinary profession. With this need in mind, a new, veterinary-specific communication and consultation skills model (WISE COACH [WC]; Appendix) was developed in 2010 at the University of California-Davis School of Veterinary Medicine with the assistance and input of numerous colleagues in private and university practice. The acronym is a reminder of the veterinarian's desired role as a coach rather than an authoritarian figure, reflecting a model of relationship-centered care, while recognizing that veterinarians have sub- 
stantial wisdom regarding patient care as a result of their extensive training and experience. Each letter of the acronym serves as a sequential guide to deliver an effective and efficient consultation: Welcome the client and patient, Investigate the history, Summarize the information obtained to this point, Explore for more details and perform a physical exam, Convey your findings, Outline a plan, Ask for feedback, Clarify expectations, and Hold both client and patient interests in mind throughout the consultation. Over the past 10 years, WC has been repeatedly revised and refined, most recently by incorporating reminders related to the Fear Free initiative, with the goal of improving consultation outcomes and increasing patient, client, and veterinarian well-being.

WISE COACH is intended to improve veterinarianclient communication and was developed, in part, in response to the growing recognition within the veterinary profession of the importance of communication skills. In a 2012-2013 survey, ${ }^{4}$ 98\% of veterinarians agreed that communication skills were as important or more important than clinical knowledge, and veterinary employers rate communication skills as one of the most important factors in selecting a new associate, ranking them after work ethic and before medical and surgical skills. ${ }^{5}$ Interpersonal communication and collaboration are well accepted as essential skills for veterinarians, ${ }^{6-9}$ and veterinary schools accredited by the AVMA Council on Education are required to include communication skills instruction in their curriculum, as the importance of training in this competency has been well documented. ${ }^{10-13}$

Proficiency in communication skills is highly reliant on the training itself. ${ }^{14}$ In the late 1990s, the CalgaryCambridge Guide (CCG) was developed as a detailed framework of specific communication skills for human medical professionals validated by research and theoretical evidence. ${ }^{15}$ While its use has been investigated in veterinary medicine ${ }^{16,17}$ and the model has been adapted to veterinary practice, the CCG has limitations in the veterinary field and was found to have a net neutral effect on veterinary student learning. ${ }^{18}$ The CCG comprises 71 individual communication skills, which can overwhelm learners, and it doesn't consider the unique triad relationship within veterinary practice of the client, veterinarian, and animal patient.

Furthermore, because few animal patients are covered by insurance, veterinarians must regularly communicate about finances with their clients, whereas human physicians rarely do so. Finally, the CCG includes process but not content skills, although there have been adaptations to try to address this. ${ }^{19}$ This can be confusing for learners, who must master both how to best communicate and what to communicate with clients. WISE COACH includes a number of helpful communication reminders from the CCG and attempts to address some of its shortcomings. But, WC is intended as a more comprehensive consultation model, rather than just a communication model; it includes other important tools and reminders designed to improve the efficacy of veterinary consultations.

The purpose of the study reported here was to assess the impact of the WC veterinary consultation model on US dog owner perceptions of veterinarians and their projected spending on veterinary care by use of a cross-sectional online qualitative and quantitative survey. It was hypothesized that use of the WC recommendations during client consultations would lead to improved client perceptions of the veterinarian (eg, an increased likelihood to return or to recommend the veterinarian to others) and facilitate client consent for recommended care, which impacts the quality of medical care provided to patients and the financial health of a practice.

\section{Materials and Methods}

\section{Participants}

A total of 1,200 unique participants were enrolled in the study. Survey participants were recruited by a market research service working with InsightsNow, a consumer behavior research firm specializing in applying the science of human psychology to understanding consumer behaviors and implicit bias. Participants were dog owners between 25 and 65 years old living in urban (35\%) or suburban (65\%) communities across the US. Dog owners living in rural communities were not included because the focus of the study was on urban and suburban dog owners. Individuals with a work history in veterinary practice or market research were also excluded. All participants reported owning 1 or more dogs and having their dog examined by a veterinarian within the prior 18 months. Female owners were targeted to make up $60 \%$ to $70 \%$ of the participants (actual percentage, 68\%) to align with research by the AVMA indicating that it is more common for women than men to be primarily responsible for dog care (85\% in 2011 and 54\% in 2016). ${ }^{20}$ Survey participants were from across the US.

\section{Survey instrument}

A 78-item survey instrument was developed in collaboration with InsightsNow (Supplementary Appendix S1). The University of California-Davis Internal Review Board administration evaluated the project and determined that further board review was not required because this was an online survey for which participation was voluntary and anonymous.

Using a single male veterinarian (JJC), female client (actor), canine patient (Miniature Australian Shepherd), and exam room, 2 mock client consultations (WC and control) for a routine ear problem were video recorded. The WC and control consultations were intended to demonstrate a typical small animal client consultation, and both followed the sequential steps of initiating the session, gathering information, performing a physical examination, explaining the findings, establishing a plan, and closing the session. The control consultation used a fairly minimalist approach, however, which did not include many of the specific additional tools suggested in the CCG or WC.

Staged video recordings of the WC and control client consultations were used, rather than video 
recordings of actual client consultations, to ensure standardization of the 2 approaches and allow for online participation by a large number of respondents. The same content questions regarding history were asked in both consultations, and the veterinarian made the same recommendations for care in both consultations. Veterinarian and client attire were the same for both consultations. In the WC consultation, however, the veterinarian incorporated WC recommendations, resulting in a somewhat longer consultation (3 minutes, 22 seconds greater duration).

Survey respondents were recruited from an online database maintained by Lucid Inc and were incentivized with a point-based system that allowed them to select among noncash rewards such as gift cards. The survey was open from May 28, 2020, to June 8,2020 . The intent of the survey was not disclosed at the outset; however, participants were informed that the survey sought their opinions on services and would require approximately 25 minutes to complete. Responses were collected via a secure survey platform that ensured no duplication of IP addresses. A monadic survey design was used whereby participants viewed one or the other video, but not both, thus avoiding comparative responses and potential bias related to video duration or which video would be viewed first. Because the survey was conducted during the COVID-19 pandemic yet showed exam room interactions without the use of masks, respondents were instructed to assume the scenario was taking place prior to COVID-19 concerns.

Eleven initial screening questions were used to ensure the desired sample of participants was obtained and that criteria for dog ownership, recent veterinary examination, gender, age, geographic location, participation in sensitive industries, and household income were met. To ensure that participants could actually see and hear the videos, equipment check questions were incorporated. Following these questions, participants answered 2 timed questions that were used to establish a baseline for assessment of implicit bias questions included later in the survey. Findings related to measurement of implicit bias are not covered in this report.

\section{Data collection}

After completing the initial screening and preparatory questions, participants were randomly assigned to view either the WC or control video. Least fill logic was used to ensure an even distribution, with the WC and control videos each viewed by 600 participants. Each video consisted of 3 segments, with questions after each section. Segment 1 consisted of the introduction and first impressions, segment 2 consisted of history taking, and segment 3 consisted of sharing of findings and treatment recommendations. A physical examination was not included to reduce the length of the video and survey.

The first video segment viewed by respondents consisted of an initial greeting by the veterinarian prior to initiation of history taking. The duration of the first segment was 69 seconds for the WC video and 9 seconds for the control video. After viewing this seg- ment, participants were asked to indicate their overall first impression of the veterinarian on a 10-point scale from very negative (1) to very positive (10).

Participants then watched the second video segment, which consisted of the veterinarian collecting a medical history, investigating the dog's ear condition, and asking broader screening questions regarding past history, preventative care, and a general body systems review. The video was paused just prior to initiation of the physical exam. The duration of this segment was 2 minutes, 5 seconds for the WC video and 1 minute, 25 seconds for the control video. After viewing this segment, participants were asked to evaluate 5 qualities of the veterinarian on a 1-10 scale (with 1 representing the lowest level of agreement and 10 representing the highest). Qualities assessed were skilled-knowledgeable, cares about me, cares about my pet, communicates clearly, and rushed-abrupt. The order of these questions was randomized. Participants were then asked to use the same 1-10 scale to indicate, on the basis of what they had seen so far, how likely they would be to follow the veterinarian's recommendations, return to see the veterinarian, and recommend this veterinarian. Question order was again randomized. Finally, the same 1-10 scale was used to assess level of satisfaction at this stage of the interaction. Participants were then asked open-ended questions of what they liked most and what they liked least at this point in the interaction, with the opportunity to respond with up to 1,200 characters of free-text entry.

Participants next watched the third and final video segment, which included the veterinarian sharing findings and recommending a plan for the ear problem. The veterinarian's recommendations for care were the same in both videos and included an ear swab and cytology, sedation and ear cleaning, and medication to go home. The duration of this video segment was 3 minutes, 14 seconds for the WC video and 1 minute, 32 seconds for the control video. Thus, the total consultation time (noting that the physical exam was not included in these videos) was 6 minutes, 28 seconds for the WC video and 3 minutes, 6 seconds for the control video.

At the conclusion of the full consultation video, participants were asked to evaluate 6 qualities of the veterinarian (the same 5 qualities rated after the second video segment and a sixth quality identified as conscientious about costs), rate their satisfaction, and indicate their likelihood to follow the veterinarian's recommendations, return to see the veterinarian, and recommend the veterinarian (again with the question order randomized). Two questions were asked regarding projected spending. Participants were asked to identify "the most you would feel comfortable paying for this office call/exam (not including any other care)," with response options ranging from "\$30 or less" to “\$100 or more" in \$10 increments. Participants were then asked, "Based on what you saw in the video, in addition to the office call/exam, which of the following would you most likely select?" with options of no treatment $(\$ 0)$; ear medication (\$30); ear swab sample and medication (\$70); sedation, ear cleaning, and medication 
(\$130); or sedation, ear cleaning, ear swab sample, and medication ( $\$ 170$ ).

Finally, participants were asked to provide a Yelp rating (based on the crowd-sourced online review platform Yelp) for the interaction with the veterinarian on a scale of 1 to 5 stars, followed by a free-text response field of up to 1,200 characters for the question, "What would you write in your review? Be as specific as possible." The final survey question captured information regarding participant ethnicity, including response options of "other" and "prefer not to say."

\section{Data analysis}

Quantitative data analyses were completed by statisticians at InsightsNow with MATLAB version R2017a (MathWorks Inc), Q version 5.9.3.0 (Q Research Software), and $\mathrm{R}$ version 3.6.3 (The R Foundation). Mean response scores were determined for all questions with a 10-point Likert-type scale. For all comparisons, values of $P \leq 0.05$ were considered significant.

For the qualitative data regarding participant perceptions of the veterinarian and Yelp reviews, responses were analyzed by an experienced coder through an inductive, iterative coding process to identify common themes. Descriptive summary statistics are reported for frequencies of categories of common perceptions.

\section{Results}

\section{Respondent demographics}

A total of 1,200 unique participants completed the survey, with all participants completing the entire survey. Of the respondents, $811(68 \%)$ were women, $386(32 \%)$ were men, and $3(<1 \%)$ were nonbinary. Among participants who watched the WC video, there were 402 (67\%) women, 196 (33\%) men, and $2(<1 \%)$ nonbinary. Among those who watched the control video, there were 409 (68\%) women, 190 (32\%) men, and $1(<1 \%)$ nonbinary.
The sample included 282 (24\%; 137 for the WC video, and 145 for the control video) Millennials (age, 25 to 39), 482 (40\%; 235 for the WC video, and 247 for the control video) Gen Xers (age, 40 to 55), and 436 (36\%; 228 for the WC video, and 208 for the control video) Boomers (age, 56 to 65). Four hundred thirty-six (39\%) participants were from the South, 257 (21\%) were from the West, 241 (20\%) were from the Northeast, and 239 (20\%) were from the Midwest. Participants from suburban communities $(n=774)$ comprised $65 \%$ of the study population, and participants from urban communities comprised 35\% (426).

Three hundred sixty-eight (31\%) participants reported an annual household income $<\$ 35,999$, 422 (35\%) reported an annual household income of $\$ 36,000$ to $\$ 80,999$, and 410 (34\%) reported a household income $\geq \$ 81,000$. Ethnicity of participants was white-Caucasian $(n=984$ [82\%]), Black-African American (72 [6\%]), Latino-Hispanic (67) [6\%]), Asian-Indian (56 [5\%]), Native American (10 [1\%]), other (8 [1\%]), and Pacific Islander (3 [<1\%]).

\section{First impression}

First impression scores assigned by participants after reviewing the first video segment were generally high for both groups but were significantly $(P \leq$ 0.001 ) higher for participants who viewed the WC video (mean score, 8.9) than for participants who viewed the control video (mean score, 8.2; Table 1).

\section{History taking}

After watching the second video segment, which consisted of the veterinarian collecting a medical history, participants rated the veterinarian significantly higher in the WC video than in the control video with regard to the qualities skilled-knowledgeable ( $P$ $\leq 0.001)$, cares about me $(P \leq 0.001)$, cares about my pet $(P \leq 0.001)$, and communicates clearly $(P \leq$ $0.001)$. The veterinarian was rated significantly $(P \leq$ $0.005)$ lower in the WC video than in the control video for the quality rushed-abrupt (Figure 1; Table 1).

Table 1-Mean \pm SD scores assigned by US dog owners in response to survey questions after viewing a video of a standardized client consultation with a veterinarian who used the approach recommended by the WISE COACH (WC) communication and consultation skills model $(n=600)$ or a minimalist approach (600; control video) to communicate with a client.

\begin{tabular}{|c|c|c|c|c|c|c|}
\hline \multirow[b]{2}{*}{ Survey question } & \multicolumn{2}{|c|}{ First impression } & \multicolumn{2}{|c|}{ History taking } & \multicolumn{2}{|c|}{ Findings and plan } \\
\hline & Control & WC & Control & WC & Control & WC \\
\hline What is your overall first impression of the veterinarian? & $8.2 \pm 1.6$ & $8.9 \pm 1.3^{a}$ & - & - & - & - \\
\hline Would you describe the veterinarian as skilled/knowledgeable? & - & - & $8.5 \pm 1.7$ & $9.1 \pm 1.3^{a}$ & $8.7 \pm 1.7$ & $9.3 \pm 1.2^{\mathrm{a}}$ \\
\hline Would you describe the veterinarian as someone who cares about me? & - & - & $7.9 \pm 2$ & $8.7 \pm 1.6^{a}$ & $8 \pm 2$ & $8.8 \pm 1.6^{a}$ \\
\hline Would you describe the veterinarian as someone who cares about my pet? & - & - & $8.5 \pm 1.7$ & $9.2 \pm 1.2^{\mathrm{a}}$ & $8.5 \pm 1.8$ & $9.3 \pm 1.2^{\mathrm{a}}$ \\
\hline Would you describe the veterinarian as someone who communicates clearly? & - & - & $8.8 \pm 1.6$ & $9.3 \pm 1.1^{\mathrm{a}}$ & $8.8 \pm 1.5$ & $9.3 \pm 1.2^{\mathrm{a}}$ \\
\hline Would you describe the veterinarian as conscientious about costs? & - & - & - & - & $7.5 \pm 2.4$ & $8.3 \pm 2.0^{a}$ \\
\hline How likely would you be to follow the veterinarian's recommendations? & - & - & $8.6 \pm 1.6$ & $9.2 \pm 1.3^{a}$ & $8.5 \pm 1.9$ & $9.1 \pm 1.6^{a}$ \\
\hline How likely would you be to return to see this veterinarian? & - & - & $8.3 \pm 1.9$ & $9.1 \pm 1.5^{\mathrm{a}}$ & $8.2 \pm 2.2$ & $9.0 \pm 1.6^{a}$ \\
\hline How likely would you be to recommend this veterinarian? & - & - & $8.2 \pm 1.9$ & $9.1 \pm 1.4^{a}$ & $8.2 \pm 2.2$ & $9.0 \pm 1.6^{a}$ \\
\hline How satisfied do you feel at this stage in the interaction? & - & - & $8.4 \pm 1.7$ & $9.1 \pm 1.3^{a}$ & $8.3 \pm 2.0$ & $9.0 \pm 1.5^{\mathrm{a}}$ \\
\hline
\end{tabular}

Participants were asked to respond to survey questions after viewing the first (initial greeting by the veterinarian prior to initiation of history taking), second (veterinarian collecting a medical history, investigating the dog's ear condition, and asking broader screening questions), and third (veterinarian sharing findings and recommending a treatment plan) segments of the video. All questions were scored on a scale from 1 (very negative or strongly disagree) to 10 (very positive or strongly agree).

a Mean score was significantly $(P \leq 0.05)$ higher for participants who viewed the WC video than for participants who viewed the control video. bMean score was significantly $(P \leq 0.05)$ higher for participants who viewed the control video than for participants who viewed the WC video.

$-=$ Not asked. 


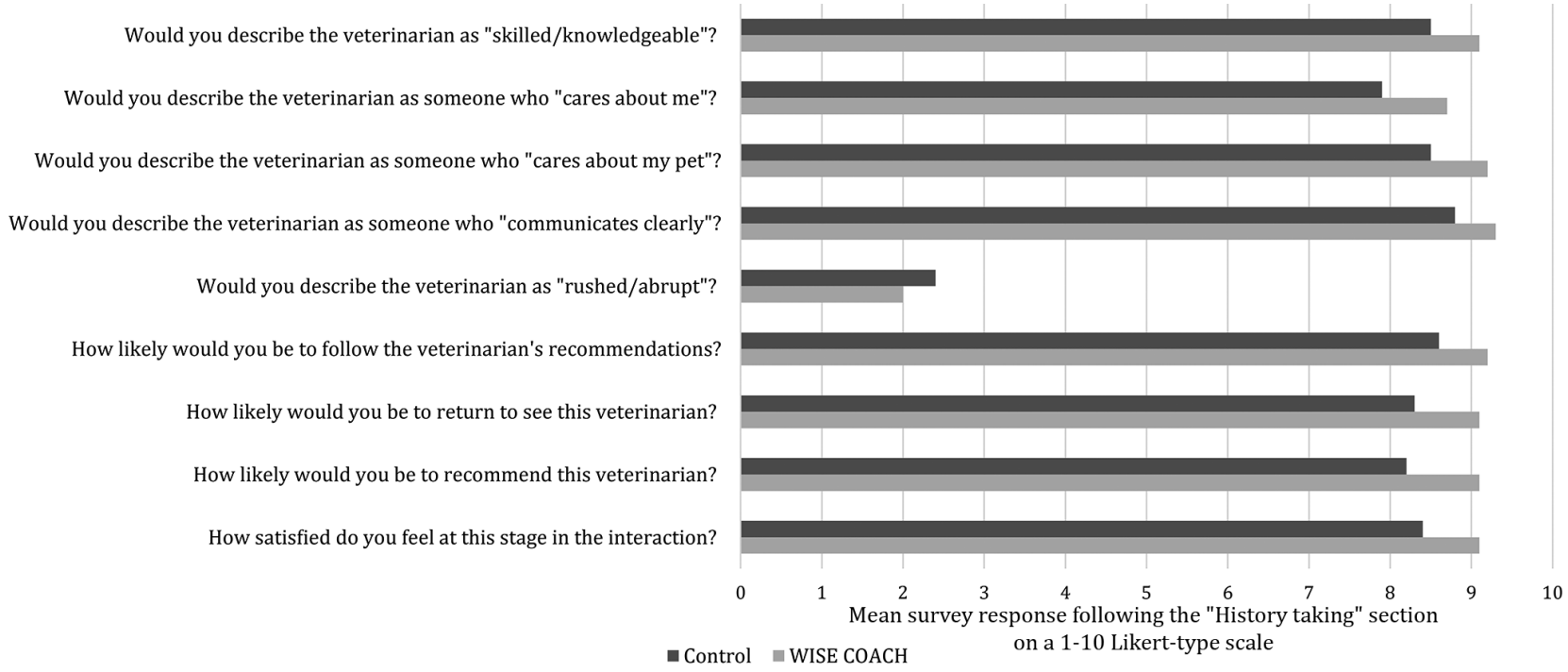

Figure 1-Mean scores assigned by US dog owners who viewed a video of a standardized client consultation with a veterinarian who used the approach recommended by the WISE COACH communication and consultation skills model ( $n$ = 600; WISE COACH video; gray bars) or a minimalist approach (600; control video; black bars) to communicate with a client. After viewing the first (initial greeting by the veterinarian prior to initiation of history taking) and second (veterinarian collecting a medical history, investigating the dog's ear condition, and asking broader screening questions) segments of the video, respondents were asked to score, on a scale from 1 (very negative or strongly disagree) to 10 (very positive or strongly agree), their reactions to various survey questions. For all questions, mean scores differed significantly $(P \leq 0.05)$ between groups.

Relative to participants who viewed the control video, those who viewed the WC video were significantly more likely to follow the veterinarian's recommendations $(P \leq$ $0.001)$, return to see the veterinarian $(P \leq 0.001)$, and recommend this veterinarian $(P \leq 0.001$; Figure 1 ; Table 1 ). Participants who viewed the WC video had significantly $(P \leq 0.001)$ higher satisfaction levels at this point in the interaction than did those who watched the control video.

For the open-ended question regarding what participants liked most at this point in the interaction, 972 comments were coded for participants who watched the WC video and

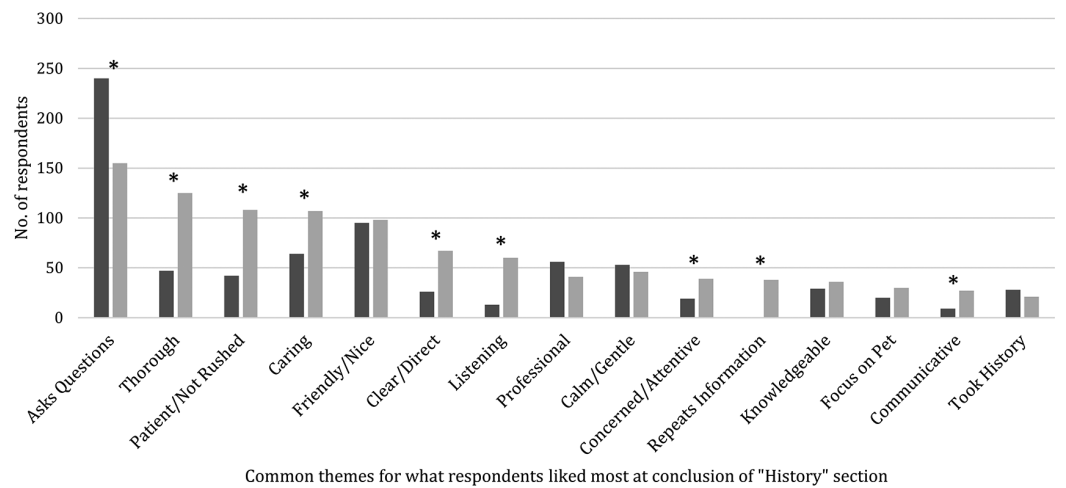

Figure 2-Common themes expressed by the participants in Figure 1 when asked what they liked most about the consultation after viewing the first 2 segments. *Frequency of this comment was significantly $(P \leq$ 0.05) different between groups. See Figure 1 for remainder of key.
680 comments were coded for par-

ticipants who watched the control video. Common themes that were identified consisted of asked questions (WC video, 155; control video, 240; $P \leq 0.001$ ), thorough (WC video, 125; control video, 47; $P \leq$ 0.001 ), patient or not rushed (WC video, 108; control video, 42; $P \leq 0.001$ ), caring (WC video, 107; control video, 64; $P=0.001$ ), friendly or nice (WC video, 98; control video, $95 ; P>0.875$ ), clear and direct (WC video, 67; control video, 26; $P \leq 0.001$ ), listening (WC video, 60; control video, 13; $P \leq 0.001$ ), professional (WC video, 41; control video, 56; $P>0.138$ ), repeats information (WC video, 38; control video, $0 ; P \leq$ 0.001 ), knowledgeable (WC video, 36; control video, 29; $P>0.444$ ), focus on pet (WC video, 30; control video, 20; $P$ > 0.193), communicative (WC video, 27; control video, 9; $P=0.004$ ), took history (WC video, 21; control video, 28; $P>0.381$ ), concerned or attentive (WC video, 39; control video, 19; $P=0.01$ ), and calm or gentle (WC video, 46; control video, 53; $P$ > 0.529; Figure 2).

For the open-ended question regarding what participants liked least at this point in the interaction, significantly $(P \leq 0.001)$ more participants who viewed the WC video responded "nothing" (n = 423) than participants who viewed the control video (341). Common themes that were identified consisted of impersonal with dog (WC video, 6; control video, 72; $P \leq 0.001$ ), too clinical or formal (WC video, 23; control video, $53 ; P \leq 0.001$ ), too thorough (WC video, 30; control video, 7; $P \leq 0.001$ ), too many 
questions (WC video, 0; control video, 13; $P \leq 0.001$ ), and veterinarian standing (WC video, 0; control video, 13; $P \leq 0.001$; Figure 3).

\section{Findings and plan}

After watching the final video segment, which consisted of the veterinarian sharing findings and recommending a treatment plan, participants rated the veterinarian significantly higher in the WC video than in the control video with regard to the qualities skilled-knowledgeable $(P \leq 0.001)$, cares about me $(P \leq 0.001)$, cares about my pet $(P \leq 0.001)$, communicates clearly $(P \leq 0.001)$, and conscientious about costs $(P \leq 0.001)$. The veterinarian was rated significantly lower in the WC video than in the control video for the quality rushed-abrupt $(P \leq 0.001$; Figure 4; Table 1).

Relative to participants who viewed the control video, those who viewed the WC video were signifi-

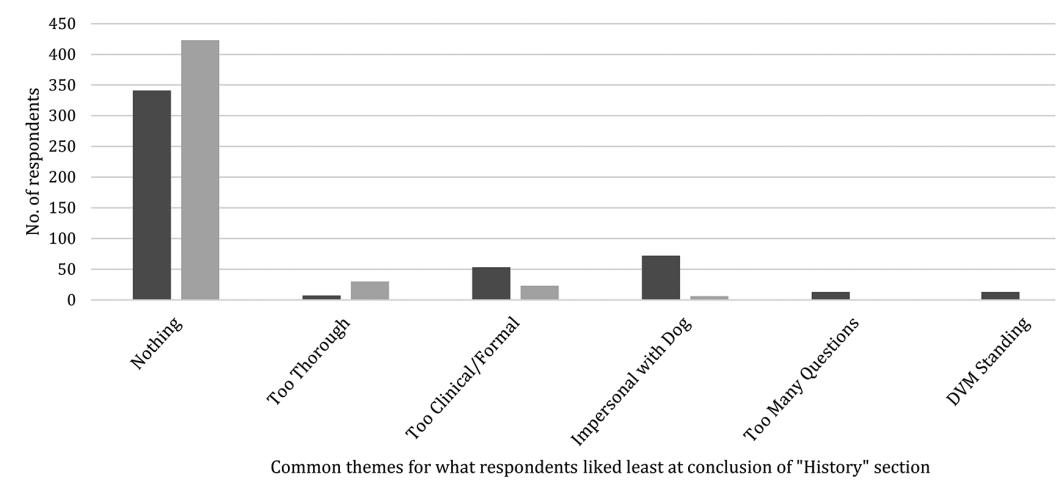

Figure 3-Common themes expressed by the participants in Figure 1 when asked what they liked least about the consultation after viewing the first 2 segments. For all themes, frequency of comments was significantly $(P \leq 0.05)$ different between groups. See Figure 1 for remainder of key. cantly more likely to follow the veterinarian's recommendations $(P \leq 0.001)$, return to see the veterinarian $(P \leq 0.001)$, and recommend the veterinarian to others $(P \leq 0.001$; Figure 4; Table 1$)$. Participants who viewed the WC video had significantly $(P \leq 0.001)$ higher satisfaction levels at this stage of the interaction, relative to those who watched the control video.

When participants were asked to indicate the most they would feel comfortable paying for this office visit, mean projected spending for participants who viewed the WC video (\$61.10) was significantly $(P \leq 0.001)$ higher than mean projected spending for participants who viewed the control video $(\$ 56.30$; Figure 5). When participants were then asked to indicate the treatment option they would most likely select, participants who viewed the WC video were significantly $(P \leq 0.001)$ more likely to choose the most thorough and expensive option (sedation, ear cleaning, ear swab sample, and medication) and significantly less likely to choose medication alone $(P \leq 0.001)$ or an ear swab sample and medication ( $P=0.004$; Figure 6) than were participants who viewed the control video. Total mean projected spending for the office visit and elected services for participants who viewed the WC video (\$186.23) was significantly $(P \leq 0.001)$ higher than total mean projected spending for participants who viewed the control video (\$161.78), representing a mean difference of $\$ 24.45$ or $15 \%$.

Finally, when participants were asked to provide a Yelp rating, the mean star rating for participants who viewed the WC video (4.7) was significantly $(P \leq 0.001)$ higher than the mean star rating for participants who

\begin{abstract}
Would you describe the veterinarian as "skilled/knowledgeable"?
Would you describe the veterinarian as someone who "cares about me"?

Would you describe the veterinarian as someone who "cares about my pet"?

Would you describe the veterinarian as someone who "communicates clearly"?

Would you describe the veterinarian as "conscientious about costs"?

Would you describe the veterinarian as "rushed/abrupt"?

How likely would you be to follow the veterinarian's recommendations?

How likely would you be to return to see this veterinarian?

How likely would you be to recommend this veterinarian?

How satisfied do you feel at this stage in the interaction?
\end{abstract}

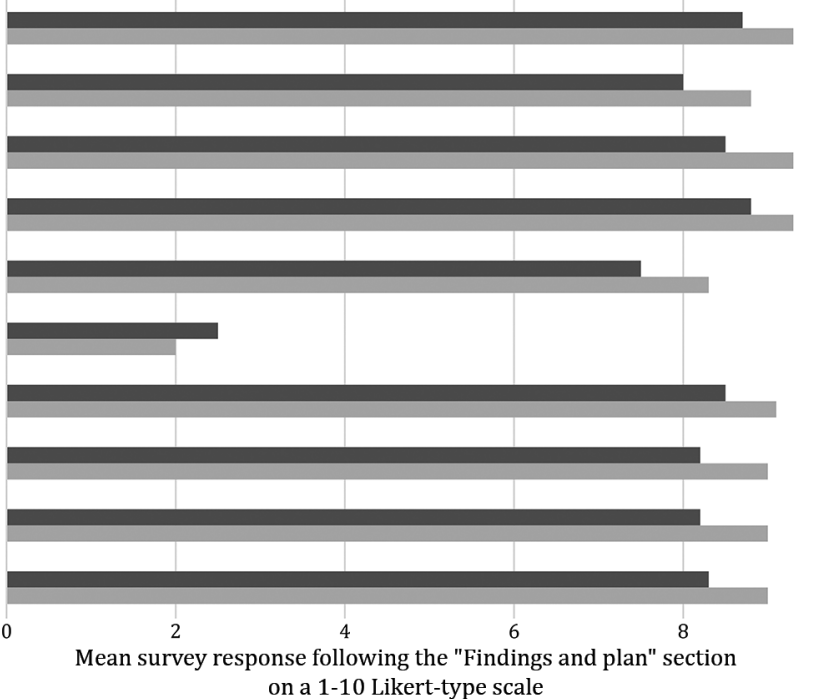

10

Figure 4-Mean scores assigned by the participants in Figure 1 after viewing all 3 segments of the consultation video (the third segment consisted of the veterinarian sharing findings and recommending a treatment plan). For all questions, mean scores differed significantly $(P \leq 0.05)$ between groups. See Figure 1 for remainder of key. 
viewed the control video (4.3). Free-text responses were coded as positive or negative, and 1,398 positive responses were identified for participants who viewed the WC video versus 998 positive responses for participants who viewed the control video. Fortyone negative responses were identified for participants who viewed the WC video versus 153 negative responses for participants who viewed the control video. Common positive themes that were identified consisted of caring (WC video, 201; control video, $120 ; P \leq 0.001$ ); thorough (WC video, 172; control video, 124; $P=0.002$ ); knowledgeable (WC video, 145; control video, 147; $P>0.946$ ); nice or friendly (WC video, 138; control video, 144; $P$ > 0.733); calm or patient (WC video, 135; control video, 88; $P=$ 0.001 ); timely, clear, and straightforward (WC video, 213; control video, 113; $P \leq 0.001$ ); professional and respectful (WC video, 102; control video, 97; $P$ $>$ 0.756); cares about dog (WC video, 96; control video, 44; $P \leq 0.001$ ); overall good (WC video, 65; control video, 49; $P>0.139$ ); and would recommend (WC video, 47; control video, $0 ; P \leq 0.001$ ). Com-

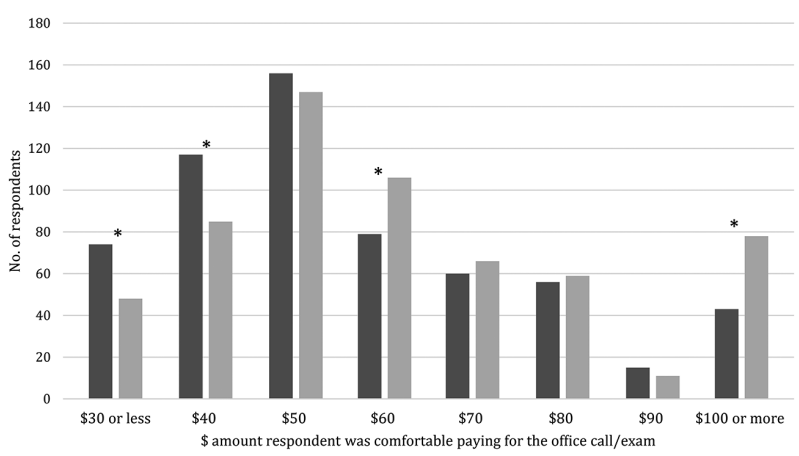

Figure 5-Frequency distributions of responses by the participants in Figure 1 when asked, after viewing the entire consultation video, the most they would feel comfortable paying for this office visit. *Frequency was significantly $(P \leq 0.05)$ different between groups. See Figure 1 for remainder of key.

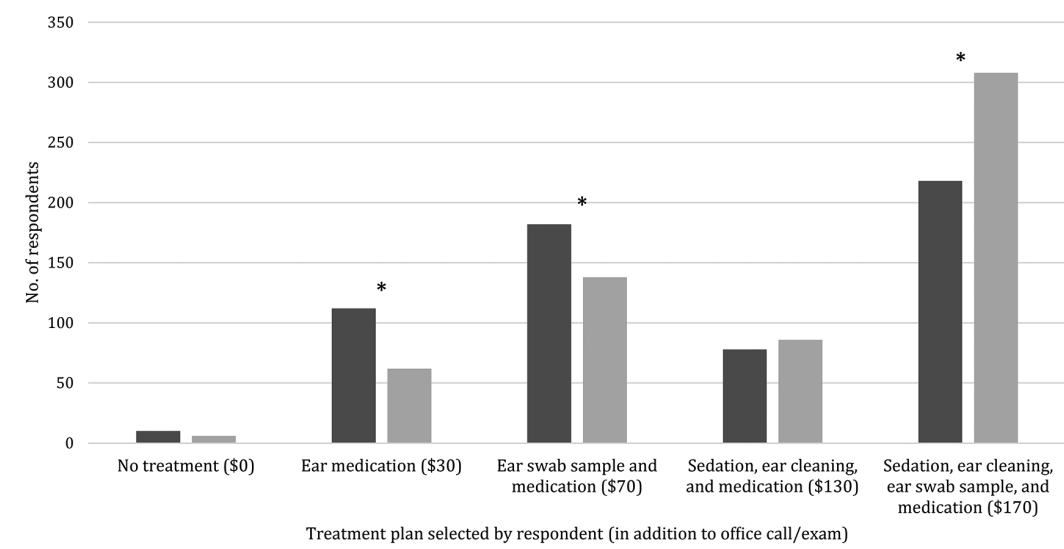

Figure 6-Frequency distributions of responses by the participants in Figure 1 when asked, after viewing the entire consultation video, which of 5 treatment options they would select. *Frequency was significantly $(P \leq$ 0.05) different between groups. See Figure 1 for remainder of key. mon negative themes that were identified consisted of expensive (WC video, 13; control video, 31; $P=$ 0.009), impersonal (WC video, 4; control video, 43; $P \leq 0.001$ ), interaction with pet (WC video, 3; control video, $21 ; P=0.001$ ), desired more options (WC video, 12; control video, $14 ; P>0.842$ ), too thorough (WC video, 7; control video, 13; $P>0.259$ ), and rushed (WC video, 0; control video, 12; $P=0.001$ ).

\section{Discussion}

The primary goal of the present study was to assess the impact of the WC veterinary consultation model on US dog owner perceptions and projected spending on veterinary care. The survey format, with staged video recordings and a large sample size, allowed for reduced variability and increased reliability of the results. The study offered compelling evidence that implementation of the WC consultation model improves client perceptions of the veterinarian, increases client compliance, and results in higher projected spending.

Although both the WC and control videos resulted in positive first impressions of the veterinarian, participants who viewed the WC video gave higher ratings. The duration of the first video segment, which consisted of the initial greeting by the veterinarian, was very short in both cases (69 seconds for the WC video and 9 seconds for the control video). The key differences between the 2 introductory approaches were that in the WC video, the veterinarian used client and patient names, greeted and complimented the patient, inquired about visit-related fear or stress, asked a nonmedical question, sat down rather than remaining standing, and explained the consultation process, including costs. Our findings suggest that these behaviors, which include being more patient-focused and relationship-centered, created a significantly more positive first impression. WISE COACH encourages veterinarians to welcome their clients and explain the process before beginning the medical history taking, sometimes referred to as the personal-before-professional approach. In the human medical field, physicians who reported a patient-centered orientation to the doctor-patient relationship were more patient-centered in their communication. ${ }^{21}$

In the history-taking portion of the WC video, the veterinarian included tools from the "ISE" portion of the WC recommendations: Investigate the history, Summarize the information obtained to this point, and Explore for more details. The 40-second difference in video length (WC video, 2 minutes, 5 seconds; control video, 1 minute, 25 seconds) was due to differences in the WC video such as asking open-ended questions, using reflective listening, and summarizing key information and client concerns, and participants who viewed the WC video rated the veterinarian significantly more highly in every quality assessed, mirroring the 
first impression findings obtained after the first video segment. Some degree of confirmation bias (ie, the tendency to interpret new evidence as confirmation of existing beliefs ${ }^{22}$ ) from first impressions may have contributed to these across-the-board differences. However, a previous study 16 also documented that asking open-ended questions, using permission statements, expressing empathy, and using reflective listening are valued by small animal clients.

Qualitative analysis of what respondents liked most and liked least after the history-taking portion of the consultation indicated that respondents placed a high value on the veterinarian asking them questions, which was the most commonly reported positive attribute for both the WC and control videos. This attribute was mentioned significantly more often by participants who viewed the control video $(n=240)$ than by participants who viewed the WC video (155), perhaps because other positive attributes took precedence for participants who viewed the WC video. This positive response to being asked questions has important implications for veterinarians in practice and supports previous research on the value of relationship-centered care that gives clients the opportunity to be active participants. ${ }^{23}$ Many respondents also appreciated that the veterinarian was thorough, patient or not rushed, caring, concerned or attentive, and clear or direct. There were no significant differences between participants who viewed the WC versus control videos in regard to the numbers of comments related to friendly or nice, professional, knowledgeable, focus on pet, took history, and calm or gentle. We believe that these findings indicate that although the veterinarian in the control video was viewed positively and equally in these dimensions, there were other important factors that led to the veterinarian in the WC video being viewed more favorably overall. Thus, it appears that perception of these qualities is perhaps necessary but not sufficient to maximize overall client perceptions.

When asked what they liked least at the conclusion of the history stage of the consultation, by far the most common response for both videos was "nothing," which was consistent with the high levels of reported satisfaction for both the WC and control videos. The most common responses regarding what participants who viewed the control video liked least were impersonal with dog and too clinical or formal, indicating the importance of veterinarians connecting with their patients and clients. A smaller number of participants who viewed the WC video reported things they liked least, with approximately $5 \%$ of respondents mentioning too thorough or too clinical or formal. This provides a helpful reminder that although some communication approaches will be successful with most clients, there is no one-size-fits-all approach.

After observing only the first 2 video segments, which made up approximately half of the client consultation, respondents in both groups indicated that they were very likely to recommend the veterinarian, return to the veterinarian, and follow the veterinarian's recommendations (even though recommendations had not yet been introduced). However, participants who viewed the WC video assigned significantly higher scores for all 3 of these questions. This suggests that clients can develop relatively strong impressions early in the consultation that may influence their subsequent decisions and actions, with the WC approach being superior to the control approach, and highlights the importance of taking a little extra time early in the client interview to build a trusting relationship with the client, which may play an important role in increased client approval of recommended care.

After observing the third and final segment of the video, which followed the physical exam and encompassed the "COACH" portion of the WC approach, respondents were asked to rate their satisfaction and likelihood to follow the veterinarian's recommendations, return, and recommend the veterinarian and to rate the veterinarian's qualities. The longer duration of this segment of the WC video (3 minutes, 14 seconds), compared with the control video (1 minute, 42 seconds) was due to inclusion of skills from the model such as determining the client's prior knowledge, describing the impact of findings on patient well-being, using visual methods to convey findings, checking the client's understanding and acceptance of the plan, and ensuring all the client's concerns had been addressed. Respondent ratings showed high marks for both groups, with significantly higher ratings across all questions from participants who viewed the WC video.

In summary, our findings suggest that veterinarians who use the WC approach are likely to be viewed more positively overall by their clients, who are both more likely to return to and recommend them. Generation of new clients and retention of existing clients are both important drivers of financial success for practices, yet little previous research has been done evaluating the financial impact of communication skills training in veterinary practice. In 1 study, ${ }^{24}$ veterinarians completing an elective communication skills training program self reported increased mean average client transaction fees. In another more recent and comprehensive study, ${ }^{14}$ however, there was no significant change in financial metrics following an in-clinic communication training program.

Discussing the financial aspects of care is not included in the CCG, which is based on research in the human medical field; this topic is, however, incorporated into the WC consultation guide. In the "Welcome" section of the model, veterinarians are reminded to outline the process of the consultation shortly after greeting the client and pet, including mentioning that costs will be discussed during the visit. An example of this approach would be, "Ms. Davis, what l'd like to do today is ask you some questions, so I understand your concerns about Lady. I'll then perform a thorough stem-to-stern physical exam and share my findings and recommendations with you, including costs to ensure we work within your budget. How does that sound?" Because cost of care is a significant concern for many clients, ${ }^{25,26}$ addressing the subject early can help put them at ease from the start, signaling that they are in control and that cost is not a taboo subject.

Following the discussion of the recommended plan in both the WC and control videos, the veteri- 
narian told the client that a technician would meet with them to review a cost estimate. The veterinarian in the WC video additionally offered to answer questions related to costs and conveyed that the client was in control of this decision. Neither video directly stated costs so that respondents could be polled to determine what plan (with associated costs) they would select. In the experience of the authors, it is often the case in actual practice that the veterinarian will make recommendations and another team member will then present the costs to the client.

An important determinant of the quality of medical care provided and financial performance of practices is the willingness of clients to follow veterinarian recommendations for care. At the conclusion of the consultation, respondents who viewed the WC video indicated they were significantly more likely to comply with the veterinarian's recommendations than did respondents who viewed the control video. In fact, $51 \%$ of respondents who viewed the WC video elected the full recommended treatment plan, compared with $36 \%$ of respondents who viewed the control video. If these findings are predictive of actual client behavior, clients working with veterinarians using the WC approach would be approximately 1.4 times as likely to approve full recommended treatment plans. Mean projected spending for the office visit among respondents who viewed the WC video (\$61.10) was 8.9\% higher than that for respondents who viewed the control video (\$56.30), and mean projected total spending for the consultation was 15\% higher for respondents who viewed the WC video versus the control video. Given that the profit margin at many veterinary practices is < 15\% and ranges from $8 \%$ to $10 \%$ for an average-performing hospital, ${ }^{27}$ this is a very relevant difference. In a practice setting, a 15\% difference in per-client average spending would have a beneficial effect on practice profitability and income of veterinarians paid via production-based compensation methods.

Although our findings offered encouraging support for the WC approach, they may underestimate the actual financial impact of this model. If clients exposed to the WC approach are more likely to identify additional medical concerns, which seems probable if they have a more positive perception of the veterinarian and are more involved in the consultation, there could be an even greater increase in medical services provided and revenue generated. This hypothesis could not be tested in this survey owing to the online standardized video study design, but further research is warranted.

An intriguing finding of the present study was that respondents indicated that the veterinarian was significantly more conscientious about costs in the WC video than the control video, even though respondents who viewed the WC video had significantly higher projected office visit and total consultation spending. This suggests that client perception of their veterinarian being cost-conscious is not directly related to projected spending. In other words, clients may spend more while also perceiving that their veterinarian is being more conscientious about costs. If increased client spending facilitates improved medical care for patients, this appears to be beneficial for the animal patient, the client, the veterinarian, and the practice.

Other elements of the WC approach are intended to address client perceptions regarding cost relative to the value of care. Veterinarians are reminded to share their findings with confidence, clarity, and sensitivity, describing the impact on patient well-being. The model recommends suggesting an optimal plan for the patient or, if that is unclear, discussing options while taking the client's lifestyle, perspective, beliefs, and abilities into consideration. Veterinarians are reminded to discuss patient comfort and explain both benefits and risks, including the risks of not proceeding. The model encourages veterinarians to confidently discuss costs and payment options before asking for the client's approval to proceed. Veterinarians are discouraged from pressuring clients, so that the client feels in control. Finally, there is a suggestion to, if appropriate, use forward booking (scheduling a procedure or the next appointment during the current visit), which has been shown to significantly improve client adherence. ${ }^{28}$ Collectively, the present research supports that these steps make a significant difference in projected approval of medical recommendations, client satisfaction, and spending.

Reputation and word-of-mouth recommendations have always been important in the veterinary profession, and today's consumers often seek guidance from online review platforms such as Yelp. According to a 2019 online survey ${ }^{29}$ of a representative sample of the US population ( $n=6,338$ ), $97 \%$ of respondents spend money on a business they find on Yelp, 90\% complete a transaction within 1 week, and $93 \%$ of respondents indicated they compared businesses before making their buying decision. With this in mind, recruitment of new clients at veterinary practices is likely to be influenced by their Yelp ratings.

Mean Yelp star ratings in the present study were significantly higher for participants who viewed the WC video (4.7) versus the control video (4.3). Because both of these are relatively high ratings on a 5-point scale, it's difficult to know whether this difference is important on a practical level. The analysis of Yelp comments, however, showed more positive comments from respondents who viewed the WC video ( $n=1,398$ ) than from respondents who viewed the control video (998). Interestingly, the numbers of comments describing the veterinarian as knowledgeable and nice or friendly were nearly equal between groups, yet respondents who viewed the WC video were much more likely to mention caring and thorough. This suggests that although a veterinarian may be perceived as both nice and knowledgeable, clients will be more satisfied and more likely to return to a veterinarian and recommend a veterinarian who has additional qualities, such as being perceived as caring and thorough. Another interesting finding was that significantly more respondents who viewed the control video 
( $n=31$ ) described the veterinarian as expensive versus those who viewed the WC video (13), even though mean spending was significantly higher for respondents who viewed the WC video.

The control video used in the present study was purposefully created to mirror the practices of veterinarians who already have fairly strong consultation skills and did score high on its own. Many practicing veterinarians, however, have never received formal communication or consultation skills training. In a 2012-2013 survey, ${ }^{4}$ for example, less than half of responding veterinarians indicated they had received communication skills training while in school or following graduation. For those who did receive training, the CCG has often served as a helpful model for teaching and learning communication skills.

WISE $\mathrm{COACH}$ includes important evidencebased communication tools from the CCG while providing a new, veterinary-specific schema with additional tools to help veterinarians meet the challenges of today's practice, which include heightened client expectations and growing financial, legal, and regulatory concerns. For example, WC reminds veterinarians to confidently discuss costs and payment options before seeking approval and to obtain informed consent by explaining benefits and possible risks, including the risk of not proceeding. The encouraging findings in this study suggest that the WC approach can provide veterinarians with sound, evidence-based guidance on conducting effective client consultations.

Although not measured in the present study, we believe that veterinarians may feel more positive or empowered when using the WC approach. Veterinary team effectiveness is known to be a driver of job satisfaction, and high effectiveness decreases cynicism and burnout. 30 Thus, the use of a consultation model that improves overall effectiveness, enriches client relations, and improves compliance and quality of care could have implications for wellness of veterinarians and hospital staff. Other studies have shown that helping or healing animals and meaningful relationships with clients are sources of veterinarian satisfaction. ${ }^{31}$ Financial limitations of owners, ethical dilemmas, and euthanasia have all been identified as sources of moral distress to veterinarians and important factors contributing to burnout. ${ }^{32,33}$ The WC approach provides targeted strategies and training to address these situations.

In considering the results of our study, it is important to recognize that survey respondents may respond differently when observing a video recording of a veterinarian-client-patient interaction versus being an active participant in one. Similarly, findings in the present study regarding client behavior, including willingness to return to the veterinarian and spending on care, were projected rather than actual. The WC video was just over 3 minutes longer than the control video, and a longer consultation may by itself foster greater satisfaction. However, in 1 study, 34 increased duration of the veterinary consultation did not result in increased client satisfaction. The additional time investment with the WC approach should be weighed against the potential benefits of improved client perception, satisfaction, referral, and compliance. Having an author of WC portray the veterinarian in the recorded videos may have introduced bias, whether conscious or unconscious. This decision was due to COVID-19 pandemic safety considerations when the videos were created. It is interesting to note, however, that there were no significant differences between open-ended responses to the WC and control videos regarding the qualities of friendly or nice, professional or respectful, knowledgeable, calm or gentle, helpful, focus on pet, or took history. Finally, the choice of a male veterinarian and female client may have introduced some form of gender bias, but both groups viewed the same veterinarian and client, and the gender composition was nearly identical between respondent groups.

The present study documented improved medical care and client compliance to veterinary recommendations with use of the WC approach. A cohesive, customized, practical, evidence-based consultation model is needed in the veterinary profession and offers benefits for patients, clients, veterinarians, and practice owners. Further research is needed to verify results in a real-time practice setting and in specific medical situations (eg, emergencies, wellness visits, and euthanasia visits). Although the WC approach has been successfully taught via a regimented program to veterinary students at the University of California-Davis School of Veterinary Medicine, further investigation is needed to establish the nature of training needed to improve the consultation skills and communication competency of veterinary practitioners. The present research opens the door for further investigation in the field of veterinary communication; such avenues might include the effect of the WC approach on veterinary wellness, the power of first impressions and implicit bias, and the specific WC tools and steps that have the biggest impact.

\section{Acknowledgments}

Funded in part by a grant from the Dr. Tom Kendall family to the University of California-Davis School of Veterinary Medicine. Third-party funding sources were not involved in the study design, data analysis and interpretation, or writing and publication of the manuscript.

Regarding conflicts of interest, Dr. Clark personally provided approximately $50 \%$ of the funding for this study. Subsequent to conducting this research, the authors formed an educational consulting service, Pet Practice Partners, that will include training opportunities on the WC model. Dr. Linder has served as a mentor on the communication training team at the University of California-Davis School of Veterinary Medicine.

The authors thank InsightsNow for their help designing, administering, and analyzing the survey and thank Derrith Lambka and Kylie Clark for their intellectual and technical assistance during this project.

\section{References}

1. Greenfield CL, Johnson AL, Schaeffer DJ. Frequency of use of various procedures, skills, and areas of knowledge 
among veterinarians in private small animal exclusive or predominant practice and proficiency expected of new veterinary school graduates. J Am Vet Med Assoc. 2004;224(11):1780-1787.

2. Robinson NJ, Dean RS, Cobb M, Brennan ML. Consultation length in first opinion small animal practice. Vet Rec. 2014;175(19):486. doi:10.1136/vr.102713

3. Boissy A, Gilligan T, eds. Communication the Cleveland Clinic Way: How to Drive a Relationship-Centered Strategy for Exceptional Patient Experience. McGraw-Hill Education: 2016.

4. McDermott MP, Tischler VA, Cobb MA, Robbé IJ, Dean RS. Veterinarian-client communication skills: current state, relevance, and opportunities for improvement. J Vet Med Educ. 2015;42(4):305-314.

5. 2017 Economic Issues Survey Report of Findings. California Veterinary Medical Association. Accessed December 18, 2020. https://cvma.net/wp-content/uploads/2017/01/ CVMAEconomicSurveyReport 2016final.pdf

6. Haldane S, Hinchcliff K, Mansell P, Baik C. Expectations of graduate communication skills in professional veterinary practice. J Vet Med Educ. 2017;44(2):268-279.

7. Roadmap for veterinary medical 4education in the 21st century: responsive, collaborative, flexible. North American Veterinary Medical Education Consortium. Accessed December 26, 2020. https://www.aavmc.org/assets/ data-new/files/NAVMEC/navmec_roadmapreport_web_ booklet.pdf

8. Hughes K, Rhind SM, Mossop L, et al. 'Care about my animal, know your stuff and take me seriously': United Kingdom and Australian clients' views on the capabilities most important in their veterinarians. Vet Rec. 2018;183(17):534. doi:10.1136/vr.104987

9. Kedrowicz AA. Clients and veterinarians as partners in problem solving during cancer management: implications for veterinary education. J Vet Med Educ. 2015;42(4):373-381.

10. Cake MA, Bell MA, Williams JC, et al. Which professional (non-technical) competencies are most important to the success of graduate veterinarians? A Best Evidence Medical Education (BEME) systematic review: BEME Guide No. 38. Med Teach. 2016;38(6):550-563.

11. Hess-Holden CL, Jackson DL, Morse DT, Monaghan CL. Understanding non-technical competencies: compassion and communication among fourth-year veterinarians-intraining. J Vet Med Educ. 2019;46(4):506-517.

12. Bachynsky EA, Dale VHM, Kinnison T, Gazzard J, Baillie S. A survey of the opinions of recent veterinary graduates and employers regarding early career business skills. Vet Rec. 2013;172(23):604.

13. Meehan MP, Menniti MF. Final-year veterinary students' perceptions of their communication competencies and a communication skills training program delivered in a primary care setting and based on Kolb's Experiential Learning Theory. J Vet Med Educ. 2014;41(4):371-383.

14. Cornell KK, Coe JB, Shaw DH, Felsted KE, Bonvicini KA Investigation of the effects of a practice-level communication training program on veterinary health-care team members' communication confidence, client satisfaction, and practice financial metrics. J Am Vet Med Assoc. 2019;255(12):1377-1388.

15. Kurtz SM, Silverman JD. The Calgary-Cambridge Referenced Observation Guides: an aid to defining the curriculum and organizing the teaching in communication training programmes. Med Educ. 1996;30(2):83-89.

16. Show A, Englar RE. Evaluating dog- and cat-owner preferences for Calgary-Cambridge communication skills: results of a questionnaire. J Vet Med Educ. 2018;45(4):534-543.

17. Shaw JR, Barley GE, Broadfoot K, Hill AE, Roter DL. Outcomes assessment of on-site communication skills education in a companion animal practice. J Am Vet Med Assoc. 2016;249(4):419-432.

18. Radford A, Stockley P, Silverman J, Taylor I, Turner R, Gray C. Development, teaching, and evaluation of a consulta- tion structure model for use in veterinary education. $J$ Vet Med Educ. 2006;33(1):38-44.

19. Kurtz S, Silverman J, Benson J, Draper J. Marrying content and process in clinical method teaching: enhancing the Calgary-Cambridge guides. Acad Med. 2003;78(8):802-809.

20. AVMA. AVMA Pet Ownership and Demographics Sourcebook: 2017-2018 Edition. AVMA Veterinary Economics Division; 2017.

21. Street RL Jr, Gordon H, Haidet P. Physicians' communication and perceptions of patients: is it how they look, how they talk, or is it just the doctor? Soc Sci Med. 2007;65(3):586-598.

22. Nickerson RS. Confirmation bias: a ubiquitous phenomenon in many guises. Rev Gen Psychol. 1998;2(2):175220.

23. Roter D. The enduring and evolving nature of the patientphysician relationship. Patient Educ Couns. 2000;39(1):5-15.

24. Kogan LR, Butler CL, Lagoni LK, Brannan JK, McConnell SM, Harvey AM. Training in client relations and communication skills in veterinary medical curricula and usage after graduation. J Am Vet Med Assoc. 2004;224(4):504507.

25. Coe JB, Adams CL, Bonnett BN. A focus group study of veterinarians' and pet owners' perceptions of the monetary aspects of veterinary care. J Am Vet Med Assoc. 2007;231(10):1510-1518.

26. Coe JB. Taming the elephant in your exam room: building confidence in discussing the cost of veterinary care while reframing how we convey value. In: Proceedings of the AAHA Connexity Conference. American Animal Hospital Association; 2020.

27. Keiser S. A balancing act: use KPIs and financial smarts to drive your practice towards higher levels or profitability. Today's Veterinary Business. Accessed January 22, 2021. https://todaysveterinarybusiness.com/a-balancing-act/

28. Forward booking; how forward booking leads to better patient care. An American Animal Hospital AssociationAmerican Veterinary Medical Association White Paper. Partners for Healthy Pets. Accessed December 23, 2020. https://www.aaha.org/globalassets/04-practiceresources/Forward-booking

29. Belt B. Study shows $97 \%$ of people buy from local businesses they discover on Yelp. Yelp Official Blog. Accessed December 26, 2020. https://blog.yelp.com/2019/10/ study-shows-97-of-people-buy-from-local-businessesthey-discover-on-yelp

30. Moore IC, Coe JB, Adams CL, Conlon PD, Sargeant JM. The role of veterinary team effectiveness in job satisfaction and burnout in companion animal veterinary clinics. J Am Vet Med Assoc. 2014;245(5):513-524.

31. Cake MA, Bell MA, Bickley N, Bartram DJ. The life of meaning: a model of the positive contributions to well-being from veterinary work. J Vet Med Educ. 2015;42(3):184-193.

32. Kipperman BS, Kass PH, Rishniw M. Factors that influence small animal veterinarians' opinions and actions regarding cost of care and effects of economic limitations on patient care and outcome and professional career satisfaction and burnout. J Am Vet Med Assoc. 2017; 250(7):785-794.

33. Kipperman B, Morris P, Rollin B. Ethical dilemmas encountered by small animal veterinarians: characterisation, responses, consequences and beliefs regarding euthanasia. Vet Rec. 2018;182(19):548.

34. Corah L, Mossop L, Dean R, Cobb K. Measuring satisfaction in the small animal consultation and its relationship to consult length. Vet Rec. 2020;187(11):446. doi:10.1136/ vr.105910

\section{Supplementary Materials}

Supplementary materials are posted online at the journal website: avmajournals.avma.org 


\section{Appendix}

Description of the WISE COACH communication and consultation skills model for conducting a client consultation in veterinary medicine.

\section{WISE COACH}

\section{Conducting Effective Client Consultations}

The WISE COACH acronym reflects a relationship where the veterinarian is an experienced, knowledgeable guide who educates and encourages clients, respecting their individuality while seeking the best outcome for the patient.

\section{Welcome the client and patient}

- Review information and adjust emotional tone to situation before entry

- Introduce self with good eye contact and thank client for their patience as needed

- Clarify client and patient names and use them throughout the interview

- Greet and compliment the patient

- Assess and ask about visit-related fear or stress; offer treats as needed

- Establish equal eye level with client (ideally sitting) and avoid barriers

- Ask nonmedical question(s) to connect on a personal level

- Explain the process and mention that cost will be discussed

- Demonstrate warmth, respect, and interest

\section{Investigate history}

- Begin with and incorporate open-ended questions

- Use attentive and reflective listening without interruption

- Solicit for other client concerns and, if necessary, establish an agenda

- Be aware of your and the client's nonverbal signals throughout the interview

- Investigate signs, severity, frequency, progression, palliation, etc

- Investigate client goals, beliefs, feelings, and concerns

- Ensure note taking, whether typed or written, is minimally intrusive

\section{Summarize information}

- Summarize key information and client concerns

- Express authentic empathy and support throughout the interview

- When appropriate, compliment the client

- Use signpost steps and transitions: "Next, l'd like to ..."

\section{Explore for more details}

- Ask about lifestyle

- Ask about past history, preventative care, diet, allergies, and behavior

- Ask about current medications and supplements

- Perform systems review

\section{[Perform exam]}

- Partner with client to minimize fear, anxiety, and stress

- Build rapport by commenting on and talking to patient

- Narrate key elements of the physical exam

\section{Convey exam findings and your thoughts}

- Share findings with confidence, clarity, and sensitivity

- Determine prior client knowledge related to findings

- Describe impact of findings on patient well-being

- Share information in manageable chunks and check client understanding

- Avoid or define medical terms

- Use visual methods to convey information

\section{Outline a plan}

- Suggest an optimal plan or, if that is unclear, describe options

- Take client's lifestyle, perspective, beliefs, and abilities into consideration

- Explain benefits and possible risks to patient, including risk of NOT proceeding

- Discuss patient comfort and goal to minimize patient distress

- Confidently discuss costs and payment options before seeking approval

- Notice and respond to nonverbal signals of concern

\section{Ask for feedback}

- Check client's understanding, feelings, and acceptance of the plan

- Help the client feel in control-avoid pressuring

- Offer alternatives as needed

- Find common ground: "We both want ..."

- Obtain and document informed consent

\section{Clarify expectations}

- Recap the plan and verify agreement

- Set realistic expectations for care and communication

- Provide written and/or online resources

- If appropriate, suggest plan to reduce patient distress on future visits

- Ask if all client concerns have been addressed

- If appropriate, schedule procedure or follow-up

Hold both client and patient interests in mind

- Act as patient advocate while respecting client perspective

- Build positive relationship with both client and patient throughout process

(c) Copyright Pet Practice Partners. Version 011221. 